\title{
Sbagliatamente? Non comune, ma corretto
}

\section{Salvatore Claudio Sgroi}

PUBBLICATO: 21 MAGGIO 2019

\section{Quesito:}

Bartolomeo B. (Lecce), Simona B. (Porto Fuori), Silvia De M. (Velletri), Sabrina N. (Bisceglie), Claudio P. (Pavia), Daniela T. (Carmignano), avendo chi sentito in TV o parlando con persone, chi letto in una e-mail un avverbio come sbagliatamente, hanno avuto il dubbio se "si può dire", "se è corretto", o se non occorra preferire la forma comune in modo sbagliato.

\section{Sbagliatamente? Non comune, ma corretto}

I

lettori di diverse regioni d'Italia (Lombardia, Lazio, Puglia) sono rimasti tutti colpiti dall'avverbio sbagliatamente, estraneo alla loro competenza attiva (ma anche a quella di chi scrive), e giunto alla loro attenzione attraverso canali diversi, orali e scritti, pubblici e privati, in bocca a nativo-italofoni ma anche a straniero-italofoni.

Queste infatti le loro testimonianze:

(i) «il banchiere Corrado Passera, nella trasmissione su La 7 Piazza Pulita ha usato, come avverbio, il termine sbagliatamente, invero assai brutto»;

(ii) "parlando con delle persone mi sono imbattuta nel termine "sbagliatamente" nel contesto di frase tipo: io sbagliatamente ho interpretato questo alle mie orecchie è suonata come orrore ma dato la persona da cui è uscito tale termine e anche documentandomi su internet mi è venuto il dubbio»;

(iii) «nella lingua parlata ho sempre sentito dire "in modo sbagliato" e ...(quasi) mai sbagliatamente". Quindi vorrei sapere se l'ultima variante è corretta».

(iv) «ho sentito a Fahrenheit una studiosa inglese di Garibaldi dire 'sbagliatamente'»;

(v) «ho ricevuto una mail che conteneva il termine 'sbagliatamente'».

La stranezza dell'avverbio sbagliatamente che ha colpito i nostri lettori (non escluso chi scrive) non riguarda la struttura morfologica del termine perfettamente regolare, trattandosi di un suffissato deaggettivale in -mente, con base aggettivale a due uscite (sbagliat-o/a) e vocale di aggiustamento in -a:

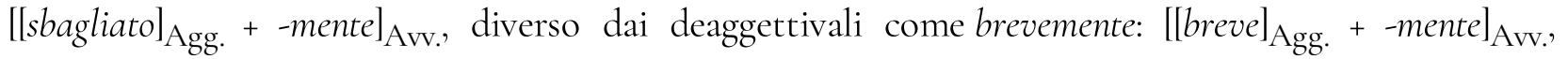
senz'alcun aggiustamento della base a una sola uscita (breve), e diverso dai deaggettivali come facilmente: $\left[\right.$ ffacile $_{\mathrm{Agg} .}+-$-mente $_{\mathrm{Avv}}$, con base in -le e conseguente cancellazione della vocale /-e/ (sulla varia problematica cfr. Grossmann-Rainer 2004, pp. 472-89; S.C. Sgroi 'Morfologi, vi esorto alla storia!' Pseudo-eccezioni nelle regole di formazione degli avverbi in -mente, in "Studi di Grammatica Italiana", vol. XXIII, 2004 [ma: luglio 2006], pp. 87-I90).

A colpire l'attenzione dei lettori è stata l'applicazione della ricordata regola di formazione dell'avverbio a questo particolare aggettivo: sbagliato, a sua volta conversione del participio passato di sbagliare. 
Una possibile restrizione semantica dell'applicazione di tale regola all'aggettivo sbagliato è da escludere vista appunto la presenza in parlanti diversi della forma sbagliatamente. La spiegazione della "stranezza" di sbagliatamente può a questo punto essere individuata nell'esistenza di sinonimi concorrenti come erroneamente, documentato col De Mauro fin dal i304-I308, o anche erratamente attestato dal I9I7, che hanno, come dire, reso difficile la vita a sbagliatamente, limitandone notevolmente la vitalità.

La rarità di sbagliatamente spiega la sua assenza non solo nella corrente lessicografia monovolume (Zingarelli 2019, De Mauro 2000, Sabatini-Coletti 2007, Devoto-Oli 2014, Treccani-Simone 2003) ma anche in quella piu ampia (Vocabolario Treccani 1997, 5 voll.; GRADIT 2007, 8 voll.), e perfino nel GDLI (I96I-2002, 2004 e 2009, 23 voll.)

A voler documentare la sua vitalità nell'uso scritto, il lettore non troverà alcun es. nella LIZ $200{ }^{4}{ }^{4}$-BIZ 20 Io (ricca di circa Iooo testi letterari dal X sec. al I936), nel PTLLIN, costituito dai romanzi Strega pubblicati nel sessantennio I947-2006, né nel domenicale del "Sole 24 Ore" (I983-2008).

Grazie a Google Libri ricerca avanzata è tuttavia possibile tracciare una storia degli usi dell'avverbio. L'esempio più antico sembrerebbe ottocentesco, prima metà: "Ma l'autorità di Torti vediamo ancora più sbagliatamente prodotta dal sig. Puccinotti in proposito delle perniciose pleuritiche" (F. G. Geromini, Dell'umano febbricitare. Nuovo saggio pratico della medicina misontologica, Milano, P. A. Molina, I84I, p. I63).

Nel 'goo citiamo un solo esempio di una nota pedagogista: "Tutte queste maniere però sono reazioni a una vita sbagliatamente compresa, e non hanno nessuna influenza modificatrice sulla vita stessa. Sono, come i divertimenti, al di fuori dell'esistenza consueta" (Maria Montessori, Il metodo della pedagogia scientifica applicato all'educazione, Roma, Maglione \& Strini, I9I8, p. IOI).

Quanto all'aspetto normativo, il giudizio dei lettori su questo suffissato è negativo, chiaramente perché estraneo alla propria competenza attiva: "invero assai brutto"; la "frase tipo: io sbagliatamente ho interpretato questo alle mie orecchie è suonata come orrore". Al punto da far nascere un "dubbio", se "si può dire", e chiedersi più volte (quasi con angoscia) se la forma "è corretta". Come dire che il diverso è ... di per sé sbagliato. Invero, a un lettore è "venuto il dubbio" che la forma potesse essere corretta, "dato la persona da cui è uscito tale termine e anche documentandomi su internet".

Per conto nostro, fermo restando che l'uso è limitato, ristretto, quasi "bloccato" dal concorrente erroneamente (ed erratamente), in considerazione degli usi illustri su ricordati e presenti all'inizio del '9oo, si tratta di una forma normativamente corretta, che sarebbe quindi sbagliato giudicare "errata" nell'uso altrui, solo perché soggettivamente valutata brutta.

\section{Cita come:}

Salvatore Claudio Sgroi, Sbagliatamente? Non comune, ma corretto , "Italiano digitale", 2019, IX, 2019/2 (aprile-giugno)

DOI: $10.35948 / 2532-9006 / 2020.3116$

\section{Copyright 2019 Accademia della Crusca}

Pubblicato con licenza creative commons CC BY-NC-ND 